\title{
In Vivo Effects of Gis-5,8,11,14,17-20:5(n-3) \\ and Cis-4,7,10,13,16,19-22:6(n-3) on Serum \\ Lipoproteins, Platelet Aggregation, and Lipid Metabolism in the Aorta of Rats
}

\author{
Nobuhiro Morisaki, Masaki Shinomiya, Nobuo Matsuoka, \\ Yasushi SaIto and Akira KumagaI \\ Second Department of Internal Medicine, School of Medicine, \\ Chiba University, Chiba 280
}

Morisaki, N., Shinomiya, M., Matsuoka, N., Saito, Y. and Kumagai, A. In Vivo Effects of Cis-5,8,11,14,17-20:5 (n-3) and Cis-4,7,10,13,16,19-22:6 (n-3) on Serum Lipoproteins, Platelet Aggregation, and Lipid Metabolism in the Aorta of Rats. Tohoku J. exp. Med., 1983, 141 (4), 397-405 — Highly purified cis$5,8,11,14,17-20: 5(n-3)$ or cis-4,7,10,13,16,19-22:6(n-3) was administered to rats for 2 weeks, and serum lipoprotein lipid levels, platelet aggregation, and lipid metabolism in the aorta in vivo were investigated. 20:5 (n-3) decreased the level of low density lipoprotein phospholipid. 22:6 (n-3) increased the level of high density lipoprotein cholesterol and decreased those of low density lipoprotein triglyceride and phospholipid. Both 20:5 (n-3) and 22:6 (n-3) markedly inhibited platelet aggreation. 20:5 (n-3) decreased the acid and neutral cholesterol esterase activities, but did not affect either acyl-CoA synthetase or acyl-CoA: cholesterol acyltransferase activity. 22:6 (n-3) had no effect on any of these enzyme activities. From these results, the roles of $20: 5(n-3)$ and $22: 6(n-3)$ in the formation of atheromatous lesions and the mechanism of the decrease in cholesterol esterase activity by $20: 5(n-3)$ were discussed. polyunsaturated fatty acid; platelet aggregation; cholesterol esterase; acyl-CoA synthetase; acyl-CoA: cholesterol acyltransferase

Greenland Eskimos have a very low incidence of ischemic heart disease. From early studies, favorable plasma lipid and lipoprotein concentrations were considered to be partly responsible for their low incidence of ischemic heart disease (Bang et al. 1971; Bang and Dyerberg 1972; Dyerberg et al. 1977). Recently, more attention has been paid to a specific polyunsaturated fatty acid, cis-5,8,11,14,17eicosapentaenoic acid (n-3) (EPA). In Greenland Eskimos the EPA concentration is high in plasma lipids (Dyerberg et al. 1975) and platelets (Dyerberg and Bang 1979). EPA inhibits aggregation of platelets in vitro (Dyerberg and Bang 1978; Whitaker et al. 1979; Needleman et al. 1979; Gryglewski et al. 1979). Greenland Eskimos have a significantly increased bleeding-time due to reduction

Received for publication, January 24, 1983.

Abbreviations used: EPA, cis-5,8,11,14,17-eicosapentaenoic acid; DHA, cis-4,7,10, 13,16,19-dochosahexaenoic acid; ACAT, acyl-CoA: cholesterol acyltransferase; HDL, high density lipoproteins; LDL, low density lipoproteins; VLDL, very low density lipoproteins. 
in platelet aggregation compared with age- and sex-matched Danish controls (Dyerberg and Bang 1979). The high amount of EPA in Greenland Eskimos is considered to be derived from their marine foods (Bang et al. 1976). Thus EPA has been suggested to be antiatherosclerotic because of its anti-aggregatory effect on platelets.

However, the diet of the Eskimos is also rich in another polyunsaturated fatty acid, i.e., cis-4,7,10,13,16,19-docosahexaenoic acid (n-3) (DHA) and monounsaturated fatty acids (C20:1, C22:1 and C24:1) (Bang et al. 1976) and consequently, the concentrations of these fatty acids are also high in their platelets (Dyerberg and Bang 1979). DHA was reported to inhibit cyclooxygenase (Needleman et al. 1976; Lands et al. 1973), and thus it may also inhibit platelet aggregation. Thus it could also be a cuase of the reduced platelet aggregation of Greenland Eskimos. Although there have been many experiments on the effect of foods rich in these $n-3$ polyunsaturated fatty acids (von Lossonczy et al. 1978; Ruiter et al. 1978; Dyerberg et al. 1980; Siess et al. 1980; Scherhag et al. 1982), the effects of purified fatty acids have not been studied because of the difficulty in obtaining large amounts of purified EPA and DHA. In addition, the effects of these fatty acids on lipid metabolism in the arterial wall have not been examined. We have suggested that lipid metabolism in the arterial wall, especially, the enzyme activities of hydrolysis and synthesis of cholesterol ester, is very important in the formation of atheromatous lesions (Shirai et al. 1980; Shinomiya et al. 1980; Morisaki and Saito 1981; Morisaki et al. 1982; Murano et al. 1982).

In the present study, purified EPA and DHA were administered to rats for 2 weeks and the effects of these fatty acids on (i) serum lipoprotein lipids, (ii) platelet aggregation and (iii) lipid metabolism in the aorta were examined because these three processes are thought to be important in development of atherosclerosis.

\section{Materials and Methods}

Chemicals. Cis-5,8,11,14,17-20:5(n-3) (98\% pure) and cis-4,7,10,13,16,19-22:6(n-3) (97\% pure) were kindly supplied by the Nippon Oil and Fat Co. (Tokyo). Cholesteryl $\left[1{ }^{14} \mathrm{C}\right]$ oleate, $\left[{ }^{1-14} \mathrm{C}\right]$ palmitate, and $\left[1-{ }^{14} \mathrm{C}\right]$ oleoyl-CoA were obtained from New England Nuclear Corp. (Boston, Mass). Triton X-100, dithiothreitol and reduced glutathione were purchased from Wako Pure Chemicals (Tokyo). Cholesterol oleate, adenosine 5'-triphosphate (ATP), coenzyme A (CoASH), oleoyl-CoA, bovine serum albumin (Fraction $\mathrm{V}$, free from free fatty acid), collagen, and adenosine $5^{\prime}$-diphosphate (ADP) were purchased from Sigma Chemical Co. (St. Louis, Mo.).

Feeding conditions. Male Wistar-King strain rats weighing about $250 \mathrm{~g}$ were given CMF chow (Oriental Kobo Co. Ltd., Tokyo) ad libitum for 2 weeks. EPA or DHA ethyl ester was administered at $100 \mathrm{mg} / \mathrm{rat}$ in $50 \mathrm{ml}$ of emulsion during the night. This emulsion was carefully prepared just before administration each evening to avoid peroxidation. The following morning the $50 \mathrm{ml}$ of emulsion was confirmed to have been consumed by each rat. During the daytime, animals had free access to water. Each group consisted of 10 animals.

Preparation of samples. Rats were starved for $18 \mathrm{hr}$ and then anesthetized with ethylether. The abdominal wall was opened and blood was collected from the inferior vena cava. Blood of $\mathbf{5}$ animals in each group was pooled for lipid analysis. Blood of the other 
5 animals in each group was used for measuring platelet aggregation. For this, 9 volume of blood was mixed with one volume of $3.8 \%$ sodium citrate in a plastic centrifuge tube, the mixture was centrifuged for $10 \mathrm{~min}$ at $250 \times \mathrm{g}$, and the upper layer was used as plateletrich plasma. Platelet-poor plasma was obtained by centrifuging the remaining contents of the tube for $20 \mathrm{~min}$ at $800 \times \mathrm{g}$. The aorta from the aortic arch to the iliac bifurcation was quickly removed and placed on ice. Enzyme solution was prepared from the aorta as reported previously (Morisaki et al. 1980).

Measurement of enzyme activities. Activities of cholesterol esterase and acyl-CoA synthetase were measured as reported previously (Shinomiya et al. 1979; Morisaki et al, 1980). Acyl-CoA: cholesterol acyltransferase (ACAT) activity was measured as follows: The reaction mixture, consisting of $25 \mu \mathrm{M}\left[1{ }^{14} \mathrm{C}\right]$ oleoyl-CoA, $2 \mathrm{mM}$ dithiothreitol, $1 \%$ bovine serum albumin, $100 \mathrm{mM}$ potassium phosphate buffer ( $\mathrm{pH} 7.4$ ), and enzyme solution in a final volume of $0.25 \mathrm{ml}$, was incubated for $15 \mathrm{~min}$ at $37^{\circ} \mathrm{C}$. The reaction was stopped by adding $2 \mathrm{ml}$ of chloroform-methanol (2:1 by vol.) and then lipid was extracted by the method of Folch et al. (1957), and the $\left[1-{ }^{14} \mathrm{C}\right]$ cholesterol oleate formed was separated by thin layer chromatography by the method of Skipski et al. (1965).

Analysis of serum lipids. Serum was fractionated by ultracentrifugation in a LP-42Ti rotor (Beckman Instruments, Inc., Cal. 92634, USA). The tube containing $175 \mu \mathrm{l}$ of serum adjusted to a density of 1.006 (tube $\mathrm{A}$ ) or 1.063 (tube $\mathrm{B}$ ) with $\mathrm{KBr}$ was centrifuged at $225,000 \times \mathrm{g}$ for $150 \mathrm{~min}$ and then the upper phase was aspirated. The remaining fraction was adjusted to its original volume by adding saline and its lipid content was measured. Tubes A and B after centrifugation and aspiration contained low density lipoprotein (LDL) and high density lipoprotein (HDL) or HDL alone, respectively. The amounts of lipoprotein fractions were calculated as follows: HDL, content of tube B; LDL, content of tube A - content of tube B; VLDL, content of whole serum - content of tube A.

Cholesterol was assayed enzymatically with cholesteryl ester hydrolase, cholesterol oxidase and peroxidase (Allain 1974). Triglyceride was measured enzymatically with lipoprotein lipase, glycerol dehydrogenase and diaphorase (Tiffany 1974). Phospholipid was assayed enzymatically with phospholipase D and choline oxidase (Takayama 1977).

Platelet aggregation. Aggregation of platelet-rich plasma was induced with $5 \mu \mathrm{M}$ ADP or $15 \mu \mathrm{g} / \mathrm{ml}$ of collagen and then measured with an aggregometer (NKK HEMA Tracel 1 model PAT-4A, Niko Bioscience, Tokyo). Platelet-poor plasma was used as control for platelet-rich plasma.

Protein measurement. Protein was measured by the method of Lowry et al. (1951).

Statistics. Statistical significances of differences between means were evaluated by Student's $t$-test.

\section{Results}

\section{Animal conditions}

The body weights of groups treated with EPA and DHA were significantly higher than that of controls, but their liver weights were not significantly different from that of controls (Table 1).

TABLE 1. Effects of 2-weeks administration of EPA and DHA on body and liver weights

\begin{tabular}{lccc}
\hline & Control group & EAP group & DHA group \\
\hline Body weight $(\mathrm{g})$ & $288 \pm 5$ & $308 \pm 3^{*}$ & $315 \pm 6^{*}$ \\
Liver weight $(\mathrm{g})$ & $8.7 \pm 0.3$ & $9.3 \pm 0.2^{\mathrm{NS}}$ & $9.2 \pm 0.3^{\mathrm{NS}}$ \\
\hline
\end{tabular}

Values are mean \pm s.E.M.

NS, not significant. $* p<0.0025$ as compared with control. 


\section{Serum lipids}

Table 2 shows the serum lipid levels of whole serum and lipoprotein fractions. The total cholesterol levels in the three groups were not significantly different. However, the HDL-cholesterol level in the DHA-treated group was slightly (10.6 $\%$ ) but significantly higher than that of controls. The LDL- and VLDL-cholesterol levels and the triglyceride level were not significantly different in the three groups. In the lipoprotein fractions, only the LDL-triglyceride level of the DHA-treated group was significantly decreased. The mean value for phospholipid in the DHAtreated group was $11 \%$ more than that of controls, but this difference was not significant. The LDL-phospholipid levels in the EPA- and DHA-treated groups were significantly lower than that of controls. The free fatty acid levels of the three groups were not significantly different.

\section{Platelet aggregation.}

The platelet counts in the three groups were not significantly different; that is, $(1.22 \pm 0.08) \times 10^{6} / \mathrm{mm}^{3}$ for the control group, $(1.22 \pm 0.28) \times 10^{6} / \mathrm{mm}^{3}$ for the EPA group, and $(1.33 \pm 0.13) \times 10^{6} / \mathrm{mm}^{3}$ for the DHA group. However, platelet aggregations stimulated by $15 \mu \mathrm{g} / \mathrm{ml}$ collagen or $5 \mu \mathrm{M} \mathrm{ADP}$ in the EPA- and

TABLE 2. Effects of EPA and DHA on serum lipid levels

\begin{tabular}{cccc}
\hline & Control group & EPA group & DHA group \\
\hline Total cholesterol $(\mathrm{mg} / 100 \mathrm{ml})$ & $74.8 \pm 2.6$ & $74.6 \pm 5.5$ & $76.0 \pm 3.4$ \\
HDL-cholesterol & $36.0 \pm 1.2$ & $38.4 \pm 1.5$ & $39.8 \pm 1.1^{*}$ \\
LDL-cholesterol & $17.0 \pm 2.5$ & $16.2 \pm 3.2$ & $17.6 \pm 3.3$ \\
VLDL-cholesterol & $21.8 \pm 4.3$ & $20.4 \pm 1.7$ & $18.2 \pm 1.7$ \\
Triglyceride $(\mathrm{mg} / 100 \mathrm{ml})$ & $65.4 \pm 7.6$ & $68.2 \pm 10.1$ & $68.0 \pm 4.3$ \\
HDL-triglyceride & $25.0 \pm 2.1$ & $25.4 \pm 2.5$ & $26.8 \pm 2.5$ \\
LDL-triglyceride & $12.4 \pm 1.3$ & $15.4 \pm 1.8$ & $7.0 \pm 0.6 \ddagger$ \\
VLDL-triglyceride & $28.0 \pm 5.1$ & $27.4 \pm 6.4$ & $34.4 \pm 2.5$ \\
Phospholipid (mg/100 ml) & $70.8 \pm 5.8$ & $73.5 \pm 4.3$ & $78.6 \pm 7.4$ \\
HDL-phospholipid & $43.6 \pm 2.1$ & $45.8 \pm 2.1$ & $49.0 \pm 3.0$ \\
LDL-phospholipid & $22.5 \pm 1.7$ & $14.5 \pm 2.3 \dagger$ & $18.6 \pm 1.4^{*}$ \\
VLDL-phospholipid & $7.4 \pm 3.0$ & $12.3 \pm 2.3$ & $9.4 \pm 2.4$ \\
Free fatty acid $(\mu \mathrm{Eq} /$ liter $)$ & $884 \pm 81$ & $988 \pm 70$ & $993 \pm 55$ \\
\hline
\end{tabular}

Values are means \pm s.e.M. $* p<0.025 ; \dagger p<0.01 ; \ddagger p<0.005$.

TABLE 3. Effects of EPA and DHA on platelet aggregation

\begin{tabular}{lcc}
\hline \multicolumn{1}{c}{ Group } & Collagen $(15 \mu \mathrm{g} / \mathrm{ml})$ & ADP $(5 \mu \mathrm{M})$ \\
\hline Control & $31.8 \pm 4.0$ & $13.4 \pm 2.2$ \\
EPA-treated & $14.0 \pm 2.1^{*}$ & $5.3 \pm 0.6^{*}$ \\
DHA-treated & $14.8 \pm 1.6^{*}$ & $5.3 \pm 0.6^{*}$ \\
\hline
\end{tabular}

Values are percentages of the maximum aggregation (mean \pm s.E.M., \%).

$* p<0.0005$. 
DHA-treated groups were significantly $(p<0.0005)$ less than that of controls (Table 3).

\section{Enzyme activities in the aorta.}

Acid and neutral cholesterol esterase activities were significantly lower in the EPA group, but not the DHA group, than in the control group (Table 4). Enzyme activities related to synthesis of cholesterol ester in the arterial wall, i.e., acyl-CoA synthetase and acyl-CoA: cholesterol acyltransferase were similar in the three groups.

TABLE 4. Effects of EPA and DHA on lipid metabolism in the aorta

\begin{tabular}{lrcr}
\hline & Control group & \multicolumn{1}{c}{ EPA group } & DHA group \\
\hline Acid cholesterol esterase & $2.91 \pm 0.46$ & $1.35 \pm 0.13^{* *}$ & $3.03 \pm 0.84$ \\
Neutral cholesterol esterase & $1.45 \pm 0.26$ & $0.72 \pm 0.17^{*}$ & $1.79 \pm 0.45$ \\
Acyl-CoA synthetase & $51.4 \pm 7.1$ & $54.8 \pm 6.8$ & $62.9 \pm 4.6$ \\
Acyl-CoA: cholesterol & $6.9 \pm 1.7$ & $5.9 \pm 0.4$ & $6.0 \pm 1.0$ \\
$\quad$ acyltransferase $\left(\times 10^{-3}\right)$ & & & \\
\hline
\end{tabular}

Values are means \pm s.E.M. (nmoles/mg protein).

$* p<0.025 ; \dagger p<0.01$.

\section{Discussion}

EPA and DHA did not have remarkable effects on the serum lipid levels in the present study (Table 2). However, the increase in the HDL-cholesterol level observed in the DHA group (Table 2) may be favorable for atherosclerosis, since HDLcholesterol was considered to be a negative risk factor of atherosclerosis (Gordon et al. 1977; Miller and Miller 1975). The significance of the decreases in the levels of LDL-triglyceride and LDL-phospholipid observed in the EPA and DHA groups in development of atherosclerosis are not yet known. Greenland Eskimos, who eat marine foods rich in EPA, DHA, and long chain mono-unsaturated fatty acids (C20:1, C22:1, and C24:1) (Bang et al. 1976) have lower plasma concentrations of total cholesterol, triglycerides, $\beta$-lipoproteins, and pre- $\beta$-lipoproteins, than ageand sex-matched Danish subjects (Bang et al. 1971; Bang and Dyerberg 1972; Dyerberg et al. 1977). Moreover, administration of a fish diet rich in EPA, DHA and long chain mono-unsaturated fatty acids to humans decreased serum total cholesterol, triglycerides and VLDL, and increased HDL-cholesterol (von Lossonczy et al. 1978). Judging from the present results, these effects of marine foods or oils cannot be explained by the effect of EPA or DHA alone; the long-term effects of long chain mono-unsaturated fatty acids such as C20:1, C22:1, and C24:1 must also be considered. The difference of species also should be taken into account.

The thrombogenic theory of atherosclerosis has been well discussed by a number of investigators (Mustard et al. 1977; Harker et al. 1978; Stemerman 1979). The low incidence of atherosclerotic diseases in Greenland Eskimos has been discussed from this point of view (Dyerberg and Bang 1978, 1979; Gryglewski et al. 1979; Needleman et al. 1979; Whitaker et al. 1979; Jorgensen and Dyerberg 1980). 
In these reports reduction of platelet aggregation in Greenland Eskimos was attributed to a high intake of EPA and a high concentration of EPA in their platelets. However, the present study showed that EPA and DHA, which are both present at high concentrations in Eskimos diets (Bang et al. 1976) reduce platelet aggregation (Table 3). Though DHA inhibits cyclooxygenase (Lands et al. 1973; Needleman et al. 1976), its effect on platelets in vitro is not yet known. This should be explored from the view point of therapy of atherosclerosis.

The significance of the LDL pathway in accumulation of cholesterol esters in cells was established by Goldstein and Brown (1982) (here only a review is cited). With respect to enzymatic metabolism of lipids, we have suggested that the balance between the synthesis and hydrolysis of cholesterol esters in the LDL pathway is related to the accumulation of cholesterol esters in the aorta (Shinomiya et al. 1980; Shirai et al. 1980; Morisaki and Saito 1981; Morisaki et al. 1982; Murano et al. 1982). In the aortas of rats, hydrolysis of cholesterol esters by cholesterol esterase shows two $\mathrm{pH}$ optima; $\mathrm{pH} 4.5$ (acid cholesterol esterase) and $\mathrm{pH} 7.5$ (neutral cholesterol esterase); these enzymes are thought to be located in lysosomes and microsomes, respectively (Shinomiya et al. 1979). For synthesis of cholesterol esters in the aorta, two enzymes are necessary; first, free fatty acid is activated by acyl-CoA synthetase and then incoporation of acyl-CoA into cholesterol is catalyzed by acyl-CoA: cholesterol acyltransferase. These enzyme activities were mainly located in microsomes in the aorta of rats (Morisaki et al. 1980; Shirai et al. 1980). In the present study, the activities of acyl-CoA synthetase and acyl-CoA: cholesterol acyltransferase were not changed by EPA or DHA administration (Table 4). However, both acid and neutral cholesterol esterase activities were significantly decreased by EPA. This suggests that EPA stimulates the accumulation of cholesterol esters both in lysosomes and microsomes if the incorporation of cholesterol esters (or LDL) is the same in the control and EPA-treated groups. DHA did not change the cholesterol esterase activity significantly (Table 4).

The mechanism of regulation of cholesterol esterase activity is not well known. In in vivo experiments, cholesterol esterase activities were decreased in tocopherol deficiency (Shirai et al. 1980; Murano et al. 1982) and in highly developed atheromatous lesions induced by hypothyroidism, vitamin $\mathrm{D}_{2}$ and high cholesterol diets (Morisaki et al. 1982). In these conditions, the membrane properties in cells of the aorta were considered to be greatly changed. As cholesterol esterase is a membrane bound enzyme (Takano et al. 1974; Shinomiya et al. 1979), its activity may well be affected by qualitative and quantitative changes in lipids. In fact, it was found that phosphatidylcholine and phosphatidylethanolamine increased the enzyme activity and sphingomyelin decreased it (Shinomiya et al. 1979). In addition the composition of the substrate mixture of the enzyme affected the enzyme activity in a very complex manner (Shinomiya et al. 1981). EPA administration may change the membrane properties of cells in the aorta and may affect the activity of cholesterol esterase. Another possibility is that EPA may affect cholesterol esterase activity by changing prostanoid metabolism. EPA 
could be a precursor of $\mathrm{PGI}_{3}$, which has a similar effect on platelet aggregation and vasodilation to $\mathrm{PGI}_{2}$ (Needleman et al. 1979; Smith et al. 1979). However, Hornstra et al. (1981) and Scherhag et al. (1982) failed to obtain any evidence for in vivo conversion of EPA to $\mathrm{PGI}_{3}$ in rats in which the EPA level was elevated by cod liver oil. On the contrary, cod liver oil feeding resulted in low $\mathrm{PGI}_{\mathbf{2}}$-like activity in the aorta of rats (Hornstra et al. 1981; Scherhag et al. 1982). Increased production of $\mathrm{PGI}_{2}$ was positively correlated with cholesterol esterase activity. It is likely that EPA administration decreased $\mathrm{PGI}_{2}$ in the aorta and decreased cholesterol esterase activity. Investigations are now in progress to clarify the mechanism regulating lipid metabolism in the serum and aorta.

\section{References}

1) Allain, C. (1974) Enzymatic determination of total serum cholesterol. Clin. Chem., 20, 470-475.

2) Bang, H.O., Dyerberg, J. \& Nielsen, A. (1971) Plasma lipid and lipoprotein pattern in Greenlandic West-Coast Eskimos. Lancet, 1, 1143-1146.

3) Bang, H.O., Dyerberg, J. \& Sinclair, H.M. (1976) The composition of food consumed by Greenland Eskimos. Acta med. scand., 200, 69-73.

4) Bang, H.O. \& Dyerberg, J. (1972) Plasma lipids and lipoproteins in Greenlandic WestCoast Eskimos. Acta med. scand., 192, 85-94.

5) Dyerberg, J., Bang, H.O. \& Hjorne, N. (1975) Fatty acid composition of the plasma lipids in Greenland Eskimos. Amer. J. clin. Nutr, 28, 958-966.

6) Dyerberg, J., Bang, H.O. \& Hjorne, N. (1977) Plasma cholesterol concentration in Caucasian Danes and Greenland West-Coast Eskimos. Dan. Med. Bull., 24, 52-55.

7) Dyerberg, J. \& Bang, H.O. (1978) Dietary fat and thrombosis. Lancet, 1, 152.

8) Dyerberg, J. \& Bang, H.O. (1979) Hemostatic function and platelet polyunsaturated fatty acids in Eskimos. Lancet, 2, 433-435.

9) Dyerberg, J., Bang, H.O. \& Aagaard, O. (1980) $\alpha$-Linolenic acid and eicosapentaenoic acid. Lancet, 1, 199.

10) Folch, J., Lees, M. \& Sloanes Stanley, G.H. (1957) A simple method for the isolation and purification of total lipids from animal tissues. J. biol. Chem., 226, 497-509.

11) Goldstein, J.L. \& Brown, M.S. (1982) The Medical Clinics of North America, edited by R.J. Havel, W.B. Saunders Co., pp. 335-373.

12) Gordon, T., Castelli, W.P., Hjortland, M.C., Kannel, W.B. \& Dawber, T.R. (1977) High density lipoprotein as a protective factor against coronary heart disease. Amer. J. Med., 62, 707-714.

13) Gryglewski, R.J., Salmon, J.A., Ubatuba, F.B., Weatheryly, B.C., Moncada, S. \& Vane, J.R. (1979) Effects of all cis-5,8,11,14,17 eicosapentaenoic acid and $\mathrm{PGH}_{1}$ on platelet aggregation. Prostaglandins, 18, 453-478.

14) Harker, L.A., Ross, R. \& Glomset, J.A. (1978) Atherogenesis: endothelial injury and paletelet-mediated intimal smooth muscle cell proliferation. In: Platelets: $A$ Multidisciplinary Approach, edited by G. de Gaetano \& S. Garattini, Raven Press, New York, pp. 89-102.

15) Hornstra, G., Christ-Hazelhof, E., Haddeman, E., Ten Hoor, F. \& Nugteren, D.H. (1981) Fish oil feeding lowers thromboxane - an prostacyclin production by rat platelets and aorta and does not result in the formation of prostaglandin B. Prostaglandins, 21. 727-738.

16) Jorgensen, K.A. \& Dyerberg, J. (1980) Platelets and atherosclerosis. A review on the role of platelets in atherosclerosis with special reference to the role of polyunsaturated 20 carbon fatty acids. Danish Med. Bull., 27, 253-259.

17) Lands, W.E.M., Le Tellier P.R., Rome, L.H. \& Vanderhoek, J.Y. (1973) Inhibition 
of prostaglandin biosynthesis. In: Advances in the Biosciences, edited by S. Bergström, \& S. Bernhard, Pergamon Press, New York, N.Y., Vol. 9, 15-28.

18) Lowry, O.H., Rosenbrough, N.J., Farr, A.L. \& Randall, R.J. (1951) Protein measurement with the folin phenol reagent. J. biol. Chem., 193, 265-275.

19) Miller, G.J. \& Miller, N.E. (1975) Plasma-high-density-lipoprotein concentration and development of ischaemic heart-disease. Lancet, 1, 16-19.

20) Morisaki, N., Matsuoka, N., Shirai, K., Saito, Y. \& Kumagai, A. (1980) Studies on lipid metabolism in rat arterial wall. Atherosclerosis, 37, 439-447.

21) Morisaki, N. \& Saito, Y. (1981) Metabolic characteristics of brain microvessels and vascular injuries. Biomedicine \& Therapeutics, 6, 21-27. (in Japanese)

22) Morisaki, N., Murano, S., Shinomiya, M., Sasaki, N., Shirai, K., Matsuoka, N., Mizobuchi, M., Akikusa, B., Saito, Y. \& Kumagai, A. (1982) Lipid metabolism in arteriosclerotic arterial wall of rats. Atherosclerosis, 43, 51-57.

23) Murano, S., Shirai, K., Matsuoka, N., Morisaki, N., Sasaki, N., Shinomiya, M., Mizobuchi, M., Saito, Y. \& Kumagai, A. (1982) Effect of tocopherol deficiency on lipid metabolism in arterial wall of spontaneously hypertensive rats on normal and high cholesterol diets. Tohoku J. exp. Med., 136, 29-34.

24) Mustard, J.F., Moore, S., Packham, M.A. \& Kinlough-Rathbone, R.L. (1977) Platelets, thrombosis and atherosclerosis. Prog. Biochem. Pharmacol., 13, 312325.

25) Needleman, P., Minkes, M. \& Raz, A. (1976) Thromboxanes: selective biosynthesis and distinct biological properties. Science, 193, 163-165.

26) Needleman, P., Raz, A., Minkes, M.F., Ferrendelli, J.A. \& Sprecher, H. (1979) Triene prostaglandins: prostacyclin and thromboxane biosynthesis and unique biological properties. Proc. nat. Acad. Sci. USA, 76, 944-948.

27) Ruiter, A., Jongbloed, W., Van Gent, C.M., Danse, L.H.J.C. \& Metz, S.H.M. (1978) The influence of dietary mackerel oil on the condition of organs and on blood lipid composition in the young growing pig. Amer. J. clin. Nutr., 31, 2195-2166.

28) Scherhag, R., Kramer, H.J. \& Dusing, R. (1982) Dietary administration of eicosapentaenoic and linolenic acid increases arterial blood pressure and suppresses vascular prostacyclin synthesis in the rat. Prostaglandins, 23, 369-382.

29) Shinomiya, M., Matsuoka, N., Shirai, K., Saito, Y. \& Kumagai, A. (1979) Studies on cholesterol esterase in rat arterial wall. Atherosclerosis, 33, 343-350.

30) Shinomiya, M., Matsuoka, N., Shirai, K., Morisaki, N., Sasaki, N., Murano, S., Saito, Y. \& Kumagai, A. (1980) Effect of panthethine on cholesterol ester metabolism in rat arterial wall. Atherosclerosis, 36, 75-80.

31) Shinomiya, M., Shirai, K., Matsuoka, N., Saito, Y. \& Kumagai, A. (1981) Studies on cholesterol ester hydrolysis in artificial lipid mixtures. Artherosclerosis, 38, 301-307.

32) Shirai, K., Matsuoka, N., Morisaki, N., Murano, S., Sasaki, N., Shinomiya, M., Saito, Y., Kumagai, A. \& Mizobuchi, M. (1980) Effects of tocopherol deficiency on lipid metabolism in the arterial wall of rats on normal and high cholesterol diets. Artery, 6, 484-506.

33) Siess, W., Roth, P., Scherer, B., Kurzmann, I., Böhlig, B. \& Weber, P.C. (1980) Platelet-membrane fatty acid, platelet aggregation and thymoboxane formation during a mackerel diet. Lancet, 1, 441-444.

34) Skipski, V.P., Smolowe, A.F., Sullivan, R.C. \& Barclay, M. (1965) Separation of lipid classes by thin layer chromatography. Biochim. biophys. Acta, 106, 386-396

35) Smith, D.R., Weatherly, B.C., Salmon, J.A., Ubatuba, F.B., Gryglewski, R.J. \& Moncada, S. (1979) Preparation and biochemical properties of $\mathrm{PGH}_{3}$. Prostaglandins, 18, 423-438.

36) Stemerman, M.B. (1979) Hemostasis, thrombosis, and atherogenesis. Atheroscler. Rev., 6, 105-146.

37) Takano, T., Black, W.J., Peters, T.J. \& De Dube, C. (1974) Assay, kinetics, and lysosomal localization of an acid cholesteryl esterase in rabbit aortic smooth muscle cells. J. biol. Chem., 249, 6723-6737. 
38) Takayama, M., Itoh, S., Nagasaki, T. \& Tanimizu, I. (1977) A new enzymatic method for determination of serum choline-containing phospholipids. Clin. chim. Acta, 79, 93-98.

39) Tiffany, K.O. (1974) Clinical evaluation of kinetic enzymatic fixed time. Clin. Chem., 20, 476-481.

40) von Lossonczy, T.O., Ruiter, A., Bronsgeest-Schoute, H.C., Van Gent, C.M. \& Hermus, R.J.J. (1978) The effect of a fish diet on serum lipids in healthy human subjects. Amer. J. clin. Nutr., 31, 1340-1346.

41) Whitaker, M.O., Wyche, A., Fitzpatrick, F., Sprecker, H. \& Needleman, P. (1979) Triene prostaglandins: prostaglandin D3 and eicosapentaenoic acid as potential antithrombotic substances. Proc. nat. Acad. Sci. USA, 76, 5919-5923. 